\title{
Eradication of intracellular Francisella tularensis in THP-I human macrophages with a novel autophagy inducing agent Hao-Chieh Chiu1 $^{1}$, Shilpa Soni ${ }^{2,3}$, Samuel K Kulp ${ }^{1}$, Heather Curry², Dasheng Wang1, John S Gunn'2,3,4, Larry S Schlesinger ${ }^{2,3,4}$ and Ching- Shih Chen*1
}

Address: ${ }^{1}$ Division of Medicinal Chemistry and Pharmacognosy, College of Pharmacy, The Ohio State University, Columbus, OH 43210, USA, ${ }^{2}$ Center for Microbial Interface Biology, The Ohio State University, Columbus, OH 43210, USA, ${ }^{3}$ Department of Molecular Virology, Immunology and Medical Genetics, The Ohio State University, Columbus, OH 43210, USA and ${ }^{4}$ Division of Infectious Diseases, Department of Internal Medicine, The Ohio State University, Columbus, OH 43210, USA

Email: Hao-Chieh Chiu - hcc6571@gmail.com; Shilpa Soni - soni.19@osu.edu; Samuel K Kulp - kulp.1@osu.edu; Heather Curry - Heather.Curry@osumc.edu; Dasheng Wang - wang.941@osu.edu; John S Gunn - gunn.43@osu.edu; Larry S Schlesinger - Larry.Schlesinger@osumc.edu; Ching-Shih Chen* - chen.844@osu.edu

* Corresponding author

\begin{abstract}
Background: Autophagy has been shown recently to play an important role in the intracellular survival of several pathogenic bacteria. In this study, we investigated the effect of a novel smallmolecule autophagy-inducing agent, AR-12, on the survival of Francisella tularensis, the causative bacterium of tularemia in humans and a potential bioterrorism agent, in macrophages.

Methods and results: Our results show that AR- 12 induces autophagy in THP-I macrophages, as indicated by increased autophagosome formation, and potently inhibits the intracellular survival of $F$. tularensis (type A strain, Schu S4) and F. novicida in macrophages in association with increased bacterial co-localization with autophagosomes. The effect of AR-12 on intracellular $F$. novicida was fully reversed in the presence of the autophagy inhibitor, 3-methyl adenine or the lysosome inhibitor, chloroquine. Intracellular F. novicida were not susceptible to the inhibitory activity of AR12 added at $12 \mathrm{~h}$ post-infection in THP-I macrophages, and this lack of susceptibility was independent of the intracellular location of bacteria.
\end{abstract}

Conclusion: Together, AR- 12 represents a proof-of-principle that intracellular $F$. tularensis can be eradicated by small-molecule agents that target innate immunity.

\section{Background}

Macroautophagy (called autophagy hereafter) is a cellular response of eukaryotic cells to a number of deleterious stimuli including nutrient deprivation, organelle damage and accumulation of unfolded proteins [1]. In addition, evidence indicates that autophagy also aids in controlling infection by certain microorganisms, including viruses, bacteria and parasites [2]. Indeed, the induction of autophagy by amino acid starvation, interferons or pharmacological agents has been shown to decrease the survival of various intracellular bacteria, including Mycobacterium tuberculosis, Group A Streptococcus pyrogenes 
and Salmonella typhimurium [3-5]. Thus, the induction of autophagy may represent a viable therapeutic approach for the treatment of infections caused by intracellular bacteria that is worthy of further investigation.

Francisella tularensis is a Gram negative, facultative coccobacillus that causes the zoonotic disease, tularemia [6,7]. Depending on the route of infection, F. tularensis can lead to different forms of tularemia. Inhalation of bacteria causes the most severe form of the disease, pneumonic tularemia, which has a mortality rate as high as 60 percent in the absence of appropriate treatment [6-8]. Because of the potential to inflict severe disease in a large number of people with an aerosolized form of the bacteria, F. tularensis is classified in Category A of potential biological warfare agents by the U.S. Centers for Disease Control and Prevention $[9,10]$. Moreover, in the absence of an U.S. Food and Drug Administration-approved vaccine and in light of the potential existence of antibiotic-resistant strains of F. tularensis created in the early 1990s [9-11], the development of new antibacterial agents with novel mechanisms against $F$. tularensis has become a priority for public safety.

In infected hosts, F. tularensis is primarily found in the macrophage [8]. After entry into macrophages by phagocytosis, F. tularensis blocks the fusion of Francisella-containing phagosomes with lysosomes and later escapes into the cytosol where it proliferates to a high number [12-14]. Subsequently, F. tularensis induces infected host cells to undergo apoptosis or pyroptosis, which leads to release of bacteria and infection of new cells $[15,16]$. In addition to this cytosolic proliferation and induction of host cell death, intracellular F. tularensis have also been found to reside in Francisella-containing vacuoles (FCVs) at later stages of intracellular infection. The FCVs are doublemembraned vacuoles, which microscopically appear similar to the autophagosmes formed during autophagy $[17,18]$. Blockage of autophagy decreased the colocalization of intracellular F. tularensis with FCVs in infected host cells $[17,19]$. Moreover, F. tularensis mutants which are incapable of escaping from phagosomes were found to be surrounded by autophagosome-like vacuoles at the early stage of intracellular infection, suggesting that autophagy could play an important role in controlling intracellular growth of Francisella within phagosomes [18,19].

In this study, we demonstrate that AR-12 (a.k.a. OSU03012), a novel small-molecule autophagy-inducing agent, is able to eradicate intracellular F. tularensis ssp. novicida (called F. novicida hereafter) and F. tularensis (type A strain Schu S4) without causing cytotoxicity to the host cells. Furthermore, inhibition of autophagosome formation and lysosomal degradation completely reversed this AR-12-induced killing of intracellular F. tularensis, indicat- ing that the anti-Francisella activity of this agent is mediated primarily through an autophagy-dependent mechanism. Together, our findings show that induction of autophagy is an effective approach for the control of intracellular F. tularensis in macrophages and suggest that AR-12 can serve as a scaffold for the development of more potent autophagy-inducing antibacterial agents.

\section{Materials and methods \\ Bacteria}

F. novicida strain U112 and F. tularensis strain Schu S4 (type A) were used throughout this study. Construction of a quadruplicate acid phosphatase mutant strain (JSG2871) of F. novicida was described in a previous study [18]. Experiments involving Schu S4 were conducted in a CDC select agent-approved BSL-3 laboratory at The Ohio State University. Bacteria were grown at $37^{\circ} \mathrm{C}$ on chocolate II agar (Becton, Dickinson and Company, Franklin Lakes, NJ) or in tryptic soy broth (TSB; Becton, Dickinson and Company) supplemented with $0.025 \%(\mathrm{w} / \mathrm{v})$ iron (III) pyrophosphate (Sigma-Aldrich, St. Louis, MO) and $0.1 \%(\mathrm{w} / \mathrm{v})$ cysteine hydrochloride (MP Biomedicals, Solon, $\mathrm{OH})$.

\section{Macrophages}

The THP-1 human monocytic leukemia cell line was maintained in RPMI 1640 medium (GIBCO-BRL, Invitrogen Corp., Carlsbad, CA) containing 10\% fetal bovine serum (FBS; GIBCO-BRL). To induce differentiation, THP1 cells were treated with $20 \mathrm{nM} 12$-O-tetradecanoylphorbol 13-acetate (TPA; Sigma-Aldrich, St. Louis, MO) for 48 h. Cells were washed twice with pre-warmed PBS and cultured with fresh RPMI 1640 medium containing 10\% FBS at $37^{\circ} \mathrm{C}$ in a humidified incubator containing $5 \% \mathrm{CO}_{2}$ prior to experimentation.

\section{Reagents and antibodies}

AR-12 was synthesized in-house as described previously with purity exceeding $99 \%$ as shown by nuclear magnetic resonance spectroscopy $(300 \mathrm{MHz})$ [20]. 3-Methyl adenine (3-MA) and chloroquine were obtained from SigmaAldrich. Stock solutions of AR-12 and chloroquine were prepared in DMSO and diluted in culture medium for treatment of cells (final concentration of DMSO, $0.1 \%$ ). 3-MA was directly dissolved in cell culture medium and freshly prepared before each experiment. The following antibodies were used in this study: anti-LC3 and anti-LC3 II (MBL, Woburn, MA); anti-Francisella tularensis ssp. novicida (ImmunoPrecise, Victoria, BC); Alexa Red-conjugated goat anti-mouse IgG and FITC-conjugated goat antimouse IgG (Invitrogen, Carlsbad, CA). 


\section{Assay for intracellular survival of Francisella in macrophages}

F. novicida and F. tularensis (type A strain Schu S4) grown overnight on chocolate II agar plates were suspended in PBS to a concentration of approximately $10^{10} \mathrm{CFU} / \mathrm{ml}$ (as estimated by an O.D. of 1.0 at $600 \mathrm{~nm}$ ). To facilitate bacterial uptake by macrophages, bacteria were opsonized with human complement by incubating in RPMI-1640 medium containing 10\% normal human serum for 30 min at $37^{\circ} \mathrm{C}$ with agitation [21]. Bacteria were added at a MOI of 50 to TPA-differentiated THP-1 macrophages seeded in 24-well plates at $2.5 \times 10^{5}$ cells/well [18]. Plates were cultured with rocking for $30 \mathrm{~min}$ at $37^{\circ} \mathrm{C}$ in a humidified incubator containing $5 \% \mathrm{CO}_{2}$, and then incubated for an additional $1.5 \mathrm{~h}$. Bacteria and macrophages were exposed to $50 \mu \mathrm{g} / \mathrm{ml}$ of gentamicin for $30 \mathrm{~min}$, followed by two washes with pre-warmed PBS to remove killed extracellular bacteria [15]. Infected macrophages were then treated in triplicate with various concentrations of AR-12 for $3 \mathrm{~h}$, after which culture medium was collected from each well and macrophages lysed with $500 \mu \mathrm{l}$ of $0.1 \%$ sodium deoxycholate in PBS at $37^{\circ} \mathrm{C}$ for $5 \mathrm{~min}$ to release intracellular bacteria [14]. Bacteria present in the collected culture medium, either as free bacteria or within unattached macrophages, were harvested by centrifugation at $16,000 \times \mathrm{g}$ for $5 \mathrm{~min}$, followed by resuspension in the macrophage lysates obtained previously. Cell lysates were serially diluted with PBS and spread onto agar plates supplemented with $0.025 \%(\mathrm{w} / \mathrm{v})$ iron (III) pyrophosphate and $0.1 \%(\mathrm{w} / \mathrm{v})$ cysteine hydrochloride, or chocolate II agar plates. CFU were calculated after incubation for $24 \mathrm{~h}$ at $37^{\circ} \mathrm{C}$. Survival of intracellular bacteria in drugtreated macrophages was calculated as a percentage of that in control (untreated) cells.

\section{Immunofluorescence microscopy}

To visualize intracellular $F$. novicida, bacteria were transformed with a green fluorescent protein (GFP) expressing plasmid (pKK214) as described in a previous study [22]. Infection of THP-1 macrophages with GFP-labeled F. novicida was performed as described above for unmodified $F$. novicida. After treatment with vehicle or AR-12, cells were washed three times with cold PBS, fixed with $4 \%$ formaldehyde (Sigma) in PBS for $20 \mathrm{~min}$ at $25^{\circ} \mathrm{C}$, and then permeabilized with $0.5 \%$ Triton $\mathrm{X}-100$ in PBS for $15 \mathrm{~min}$ followed by blocking with $3 \%$ bovine serum albumin (BSA) in PBS overnight at $4^{\circ} \mathrm{C}$. After three washes with PBS, infected macrophages were incubated with primary antibody in PBS containing 1\% BSA for $1 \mathrm{~h}$ at $25^{\circ} \mathrm{C}$ and then with Alexa Red- or FITC-conjugated secondary antibody for $1 \mathrm{~h}$ at $25^{\circ} \mathrm{C}$. Macrophage nuclei were stained with 4'-6-diamidino-2-phenylindole (DAPI) contained in the Vectashield Mounting Medium (Vector Laboratories, Burlingame, CA). The slides were examined using a Nikon TE300 wide-field fluorescent microscope equipped with a digital camera (CoolSnap HQ, Roper Scientific, Tucson, AZ) or a Zeiss LSM 510 confocal laser scanning microscope system. To assess co-localization of intracellular bacteria with autophagosomes, three-dimensional images acquired by confocal microscopy were utilized to ensure the direct contact of bacteria with autophagosomes.

\section{Immunoblotting}

Cells were washed with cold PBS, then suspended in MPER protein extraction reagent (Pierce, 7 Rockford, IL), vortex vigorously and incubated on ice for $10 \mathrm{~min}$. After centrifugation at $14,000 \times \mathrm{g}$ for $15 \mathrm{~min}$ at $4^{\circ} \mathrm{C}$, the lysate supernatants were mixed with $4 \times$ Laemmli buffer and incubated at $95^{\circ} \mathrm{C}$ for $10 \mathrm{~min}$. Equivalent amounts of total protein were resolved on a SDS-acrylamide gel (20 $\mu \mathrm{g} / \mathrm{lane}$ ) and transferred to $0.2 \mu \mathrm{m}$ nitrocellulose membranes (Gelman, Pall Corp., East Hills, NY). The membranes were blocked with 3\% skim milk in TBS for $30 \mathrm{~min}$ and then washed twice with $0.5 \%$ Tween-20 in TBS (TBST). The membrane was incubated with primary antibody at the appropriate dilution in TBST for $12 \mathrm{~h}$ at $4^{\circ} \mathrm{C}$, washed three times with TBST, incubated with HRP-conjugated goat IgG secondary antibody in TBST containing $1 \%$ skim milk for $2 \mathrm{~h}$, and then washed three times with TBST. The immunopositive bands were visualized by enhanced chemiluminescence (GE Amersham, Piscataway, NJ]) followed by exposure of X-ray film (Hyperfilm, GE Amersham). Quantification of the density of bands was performed using Gel-Pro Analyzer (V3.1, Media Cybernetics, Bethesda, MD).

\section{Macrophage viability assay}

The effect of AR-12 on macrophage viability was assessed by using the 3-(4,5-dimethylthiazol-2-yl)-2,5-diphenyltetrazolium (MTT) assay [23]. THP-1 macrophages were seeded into 96-well plates at $2.5 \times 10^{4}$ cells/well (minimum of six wells per test group) in RPMI 1640 medium supplemented with $10 \%$ FBS, and then incubated overnight at $37^{\circ} \mathrm{C}$ in a humidified incubator containing $5 \% \mathrm{CO}_{2}$. The medium from each well was removed and replaced with fresh $10 \%$ FBS-supplemented RPMI 1640 medium containing various concentrations of AR-12. Controls received DMSO alone at a concentration equal to that in drug-treated cells. After $3 \mathrm{~h}$ of treatment, the medium was removed, replaced by $100 \mu \mathrm{l}$ of $0.5 \mathrm{mg} /$ $\mathrm{ml}$ of MTT in $10 \%$ FBS-containing medium, and the cells were incubated at $37^{\circ} \mathrm{C}$ for $30 \mathrm{~min}$. Medium was removed from each well, and the reduced MTT dye was solubilized in $100 \mu \mathrm{l} /$ well of DMSO. Absorbance at $570 \mathrm{~nm}$ was determined on a plate reader. The viability of drug-treated cells was calculated as a percentage of vehicle-treated control cells, and an $\mathrm{IC}_{50}$ for cell viability was determined by using CalcuSyn software (Biosoft, Cambridge, UK). In addition to the MTT assay, the effect of AR-12 on the viability of F. novicida-infected THP-1 macrophages was con- 
firmed by the lactate dehydrogenase (LDH) release assay [24] using the CytoTox $96^{\circledR}$ Non-Radioactive Cytotoxicity Assay kit (Promega, Madison, WI, USA).

\section{Extracellular bacterial growth assay}

F. novicida grown overnight on a chocolate II agar plate were suspended in PBS to an O.D. of 1.0 at $600 \mathrm{~nm}$, which was equivalent to $10^{10} \mathrm{CFU} / \mathrm{ml}$, and then diluted in modified TSB to a final concentration of $10^{4} \mathrm{CFU} / \mathrm{ml}$. The bacterial suspension was exposed to various concentrations of AR-12 in triplicate in 96-well plates. Bacterial growth in each well was monitored spectrophotometrically on a microplate reader (Molecular Devices, Sunnyvale, CA) at $37^{\circ} \mathrm{C}$ and a wavelength of $600 \mathrm{~nm}$ with readings taken every $30 \mathrm{~min}$ for $8 \mathrm{~h}$.

\section{Statistical analysis}

Data are expressed as means \pm SD. Group means were compared using a two-tailed $t$-test for independent samples. Differences were considered significant at $P<0.05$. Statistical analyses were performed using SPSS for Windows (Version 16.0; SPSS, Inc. Chicago, IL).

\section{Results \\ AR-I 2 induces autophagy in human macrophages without causing cytotoxicity}

AR-12 is an orally bioavailable small-molecule inhibitor of phosphoinositide-dependent kinase (PDK)-1 that was derived by structural modification of the cyclooxygenase2 (COX-2) inhibitor, celecoxib, but is devoid of COX-2inhibitory activity [20]. In addition to PDK-1 inhibition, recent findings indicate that AR-12 is capable of inducing autophagy in a variety of cells at concentrations of $1-5$ $\mu \mathrm{M}$ [25-27]. Importantly, we recently showed that AR-12 caused the clearance of intracellular Salmonella Typhimurium, in part, through this autophagy-inducing activity [28]. To assess whether AR-12 can induce autophagy in human macrophages, THP-1 macrophages were exposed to $1 \mu \mathrm{M}$ of AR-12, and then evaluated for evidence of autophagy by immunocytochemistry and Western blot analysis. First, the formation of autophagosomes in the cytosol was visualized by immunofluorescent staining with antibody against microtubule-associated protein light chain 3-II (LC3-II), a specific autophagosome marker. During autophagy, the cytoplasmic form of the protein, LC3-I, is cleaved and recruited to the autophagophore, where LC3-II is generated via site-specific lipidation. As shown in Fig. 1A, AR-12 induced a transient increase in the number and size of autophagosomes in the cytosol of human macrophages that peaked at $60 \mathrm{~min}$ after drug exposure. Second, immunoblotting of LC3 protein in AR-12-treated macrophages also showed a transient increase in LC3-II levels (Fig. 1B). This fluctuation in the presence of autophagosomes and LC3-II in AR-12treated macrophages indicated that autophagy was acti- vated and that the subsequent lysosomal degradation of autophagosomes was unaffected. Although excessive autophagic activity can lead to cell death, no cytotoxic effect of AR-12 on THP-1 macrophages was observed after $3 \mathrm{~h}$ of treatment with concentrations of up to $10 \mu \mathrm{M}$ (Fig. 1C). Together, these findings indicate AR-12 is capable of inducing autophagy in THP-1 macrophages at a concentration that exhibits no cytotoxicity.

\section{AR-I 2 inhibits intracellular survival of Francisella tularensis in human macrophages}

To examine whether the autophagy induced by AR-12 is associated with an increase in colocalization of intracellular F. novicida with autophagosomes, cells infected with GFP-labeled $F$. novicida were treated with vehicle or $1 \mu \mathrm{M}$ of AR-12 for $60 \mathrm{~min}$ and then probed with LC3-II antibody. The colocalization of bacteria with LC3-II-positive puncta was determined by confocal fluorescence microscopy. Data indicated that approximately $15 \%$ of $F$. novicida in AR-12-treated THP-1 macrophages were colocalized with LC3-II-positive puncta, compared to 5\% in vehicle-treated macrophages after $1 \mathrm{~h}$ of AR-12 treatment (data not shown). To examine whether this increase in bacteria-autophagosome colocalization is associated with reductions in the intracellular survival of $F$. novicida, infected THP-1 macrophages were exposed to different concentrations of AR-12 for $3 \mathrm{~h}$, and then the number of surviving intracellular bacteria was assessed by CFU counts. As shown in Fig. 2A, the survival of intracellular $F$. novicida was significantly reduced by AR-12 at concentrations $\geq 1 \mu \mathrm{M}$. As AR-12 has not been shown to induce autophagy at sub- $\mu \mathrm{M}$ concentrations, these data indicate a correlation between autophagy induction and inhibition of intracellular bacterial survival in AR-12-treated THP-1 macrophages. In addition to $F$. novicida, the effect of AR-12 on intracellular F. tularensis (type A strain Schu S4) was also examined. With exposure to various concentrations of AR-12 for $3 \mathrm{~h}$, the survival of intracellular Schu S4 in THP-1 macrophages decreased in a dose-dependent manner (Fig. 2B). This AR-12-induced intracellular bacterial killing, however, was not attributable to the death of infected host cells since AR-12 had no appreciable effect on the viability of bacteria-infected THP-1 macrophages after $3 \mathrm{~h}$ of treatment with concentrations up to $10 \mu \mathrm{M}$ (Fig. 2C). A similar lack of cytotoxicity was also observed at 6 and $12 \mathrm{~h}$ of treatment with $5 \mu \mathrm{M}$ of AR-12 (data not shown). Confirmatory assays using LDH release as an indicator of cytotoxicity revealed a similar lack of effect on the viability of infected THP-1 macrophages (data not shown). Furthermore, to determine whether this AR-12induced clearance of intracellular Francisella from macrophages involved a direct action of the drug on bacteria, $F$. novicida were exposed to AR-12 during growth in modified TSB. Figure 2D shows that AR-12 exhibited no direct inhibitory effect on the growth of $F$. novicida, suggesting 
A

$1 \mu \mathrm{M}$ AR-12 Exposure Time (min)

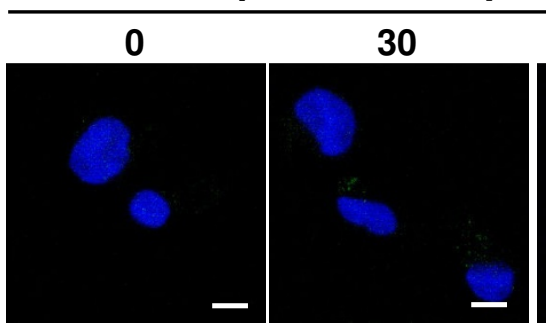

B

$1 \mu \mathrm{M}$ AR-12

Exposure Time (min)

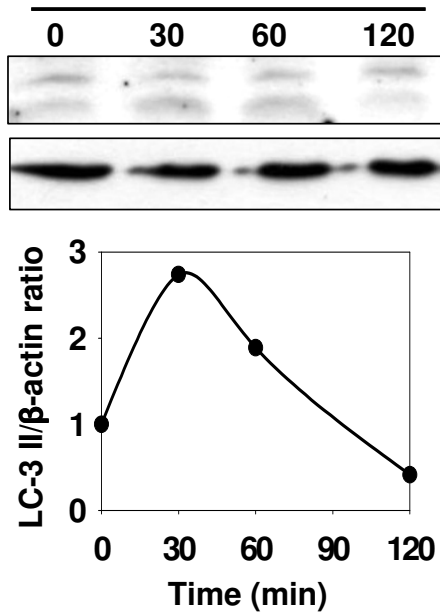

120

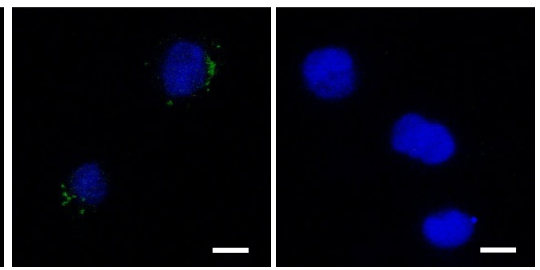

C

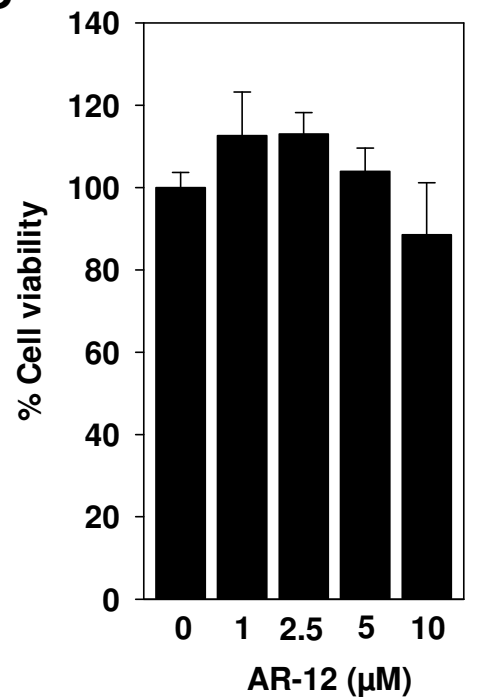

Figure I

Induction of autophagy in THP-I macrophages by AR-I2. (A) AR-I2 induced autophagosome formation in uninfected THP-I macrophages. THP-I cells were induced to differentiate by treatment with I2-O-tetradecanoylphorbol I3-acetate and then treated as indicated. Autophagosomes were visualized by immunofluorescence microscopy after staining with antibody against LC3-II. Scale bar, $10 \mu \mathrm{m}$. (B) AR-I2 induced the accumulation of LC3-II in uninfected THP-I macrophages. Above: Western blot analysis of the time-dependent effect of AR-I 2 on LC3-II formation in uninfected THP-I macrophages. $\beta$-Actin was used as a loading control. Below: The ratio of LC3-II/ $\beta$-actin in the blot after different time of AR-12 exposure (C) AR-I2 did not affect the viability of uninfected THP-I macrophages. Cells were treated with the indicated doses of AR-I2 for $3 \mathrm{~h}$ and cell viability was assessed by the MTT assay. Points, mean; bars, \pm SD $(n=6)$.

that the inhibition of intracellular survival of Francisella is mediated indirectly via effects on host cells. Together, these findings show that AR-12 is capable of inhibiting growth of both human-virulent and human-avirulent subspecies of F. tularensis in human macrophages through a host cell-directed mechanism.

\section{The inhibitory activity of AR- 2 against intracellular Francisella is autophagy-dependent}

To verify the role of autophagy in AR-12-induced killing of intracellular Francisella, we assessed the effect of blocking autophagy on the anti-Francisella activity of AR-12. 3MA suppresses autophagy through inhibition of class III phosphatidylinositol-3-kinase (PI3K), the enzyme that catalyzes the production of phosphoinositide-3-phosphate, which plays an important role in the formation of autophagosomes. Treatment of infected THP-1 macrophages with 3-MA reversed the inhibitory effect of AR-12 on the survival of intracellular F. novicida (Fig. 3A).

At late stages of the autophagic process, autophagosomes fuse with lysosomes to form autolysosomes, the contents of which are subsequently degraded by lysosomal enzymes. Consequently, to examine whether the blockage of lysosomal degradation will affect AR-12's anti-Francisella activity, we utilized chloroquine, an inhibitor of lysosomal degradation, to block the degradation of autophagosomal contents. As shown, the reduction of 
A
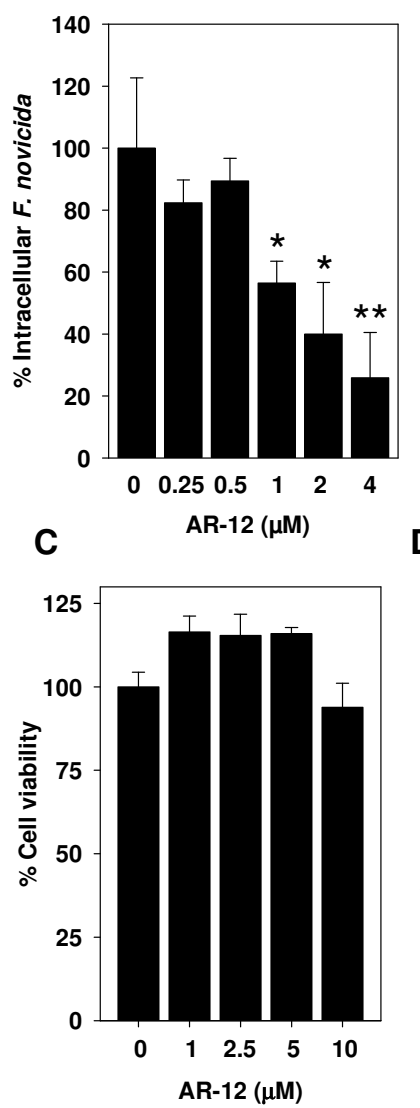

B
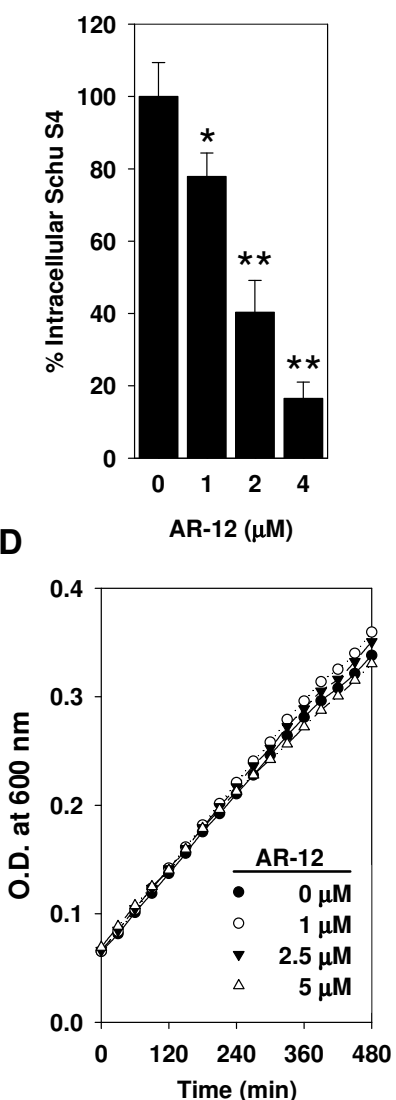

Figure 2

Inhibition of intracellular survival of $F$. tularensis in THP-I macrophages by AR- I 2. (A) Dose-dependent effect of AR-I2 (3 h treatment) on the intracellular survival of F. novicida in THP-I macrophages. Post-treatment CFU values are expressed as a percentage of those in the control group. Columns, mean; bars, $\pm S D(n=3)$. $* P<0.05$, **P< 0.01 . (B)

Dose-dependent effect of AR- 12 (3 h treatment) on the intracellular survival of $F$. tularensis (a type A strain, Schu S4) in THP-I macrophages. Points, mean; bars, \pm SD $(n=3)$. $* P<$ 0.05 , $* * P<0.01$. (C) AR-12 did not show cytotoxic effect on the viability of $F$. novicida infected THP-I macrophages. Cells infected with $F$. novicida and treated with the indicated doses of AR-I 2 for $3 \mathrm{~h}$. Cell viability was assessed by the MTT

assay. Points, mean; bars, \pm SD $(n=3)$. (D) Effect of AR- 12 on the growth of $F$. novicida in modified TSB broth. Points, mean; bars, $\pm \operatorname{SD}(n=6)$.

A

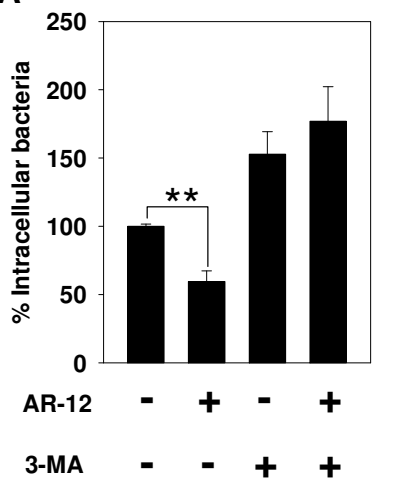

B

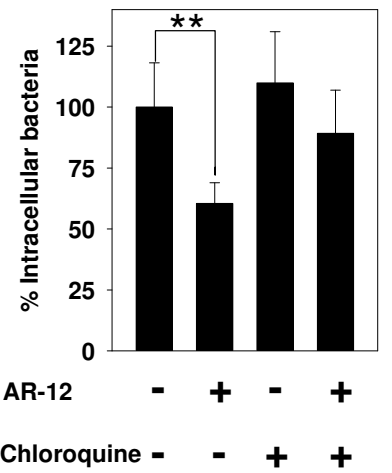

Figure 3

Inhibition of the intracellular survival of $F$. novicida in THP-I macrophages by AR-I 2 involves an autophagy-dependent mechanism. (A) The autophagy inhibitor 3-MA prevented AR-12-induced inhibition of intracellular bacterial survival. THP-I macrophages were infected with $F$. novicida and then treated for $3 \mathrm{~h}$ with $2 \mu \mathrm{M}$ AR-I 2 or DMSO in the presence and absence of 10 mM 3-MA as indicated. The number of surviving intracellular bacteria was determined and expressed as a percentage of that in the DMSO only group. Points, mean; bars, \pm SD $(n=3)$. **P< 0.0I. (B) Chloroquine, an inhibitor of lysosomal degradation, prevented AR-12-induced inhibition of intracellular bacterial survival. THP-I macrophages were infected with $F$. novicida and then treated for $3 \mathrm{~h}$ with $2 \mu \mathrm{M}$ AR-12 or DMSO in the presence and absence of $10 \mu \mathrm{M}$ chloroquine as indicated. The number of surviving intracellular bacteria was determined and expressed as a percentage of that in the DMSO only group. Points, mean; bars, $\pm S D(n=3)$. $* * P<0.01$.

intracellular $F$. novicida induced by AR- 12 was completely reversed in the presence of $10 \mu \mathrm{M}$ of chloroquine (Fig. 3B). Together, these findings indicated that autophagy plays a major role in the eradication of intracellular $F$. tularensis by AR-12.

\section{The susceptibility of intracellular Francisella to AR-I2 varies at different time points post-infection}

During the course of intracellular infection of macrophages, F. tularensis is located within different cellular compartments including the phagosome from which it escapes, the cytosol where it proliferates, and, at later stages, the FCVs. Mohapatra et al. have demonstrated that during infection of THP-1 human macrophages with $F$. novicida, $98 \%$ and $60 \%$ of intracellular bacteria reside in the vacuoles at $2 \mathrm{~h}$ and $24 \mathrm{~h}$ post-infection, respectively, while at $12 \mathrm{~h}$ post-infection, only about $1 \%$ are present in vacuoles as the vast majority of bacteria have escaped to the cytosol [18]. To assess whether the inhibitory activity of AR-12 on the intracellular survival of $F$. novicida varies with the intracellular location of bacteria, infected THP-1 
macrophages were treated with various doses of AR-12 for $3 \mathrm{~h}$ starting at 2.5, 12 and $24 \mathrm{~h}$ post-infection, and the surviving intracellular bacteria were enumerated by the CFU assay. While AR-12 showed potent dose-dependent inhibitory activity on intracellular F. novicida at $2.5 \mathrm{~h}$ and $24 \mathrm{~h}$ post-infection, no significant inhibition of intracellular survival was observed at $12 \mathrm{~h}$ post-infection (Fig. 4). This result indicates a lack of inhibitory activity against bacteria at $12 \mathrm{~h}$ post-infection, and that AR-12's antibacterial activity might be specific to Francisella enclosed within intracellular vacuoles.

To verify whether the intracellular location affects the susceptibility of intracellular Francisella to AR-12, the effect of AR-12 on a quadruplicate acid phosphatase deficient strain $(\triangle \mathrm{ABCH})$ of $F$. novicida was examined. The combined deletion of four acid phosphatases (A, B, C and $\mathrm{H}$ ) of $F$. novicida has been shown to impair the ability of bacteria to escape the phagosome as these bacteria were demonstrated to always reside in a vacuolar compartment in THP-1 macrophages at 2, 12 and 24 h post-infection [18]. However, as shown in Figure 4, whereas the intracellular $\triangle \mathrm{ABCH}$ mutant was susceptible to AR-12 at $2.5 \mathrm{~h}$ and 24 $\mathrm{h}$ post-infection, it was resistant at $12 \mathrm{~h}$ post-infection comparable to the wild type strain, indicating that the resistance of intracellular $F$. novicida to AR-12 at 12 h postinfection is not due to the intracellular location of bacteria but rather to another mechanism.

\section{Discussion}

Recently, the concept of boosting host defense mechanisms against intracellular pathogens has received wide attention in the infectious disease arena [29]. From a therapeutic perspective, targeting host immunity with an orally bioavailable small-molecule agent represents a novel strategy for antimicrobial therapy. Here we present findings that provide proof-of-principle of the feasibility of treating Francisella infection by targeting autophagy in phagocytes with a small-molecule agent. Our results show that AR-12 is a potent inhibitor of the intracellular survival of $F$. tularensis and activator of autophagy in macrophages at concentrations that induce no cytotoxicity in host macrophages. Although AR-12 has been shown to be cytotoxic to cancer cells, the antibacterial effects of AR-12 occur at lower concentrations and after shorter treatment durations. Moreover, it is worth noting that our previous in vivo evaluations of AR-12 in murine models of cancer revealed that continuous treatment with AR-12 were well tolerated and induced no dose-limiting toxicities [30,31] in association with plasma concentrations of approximately $2.5 \mu \mathrm{M}$ (Chen, unpublished data). These findings suggest that toxicities associated with the levels of AR-12 needed for antibacterial effects, if they occur, will be minimal.

Several intracellular bacteria, including Shigella, Legionella and Burkholderia, are capable of evading autophagic erad-

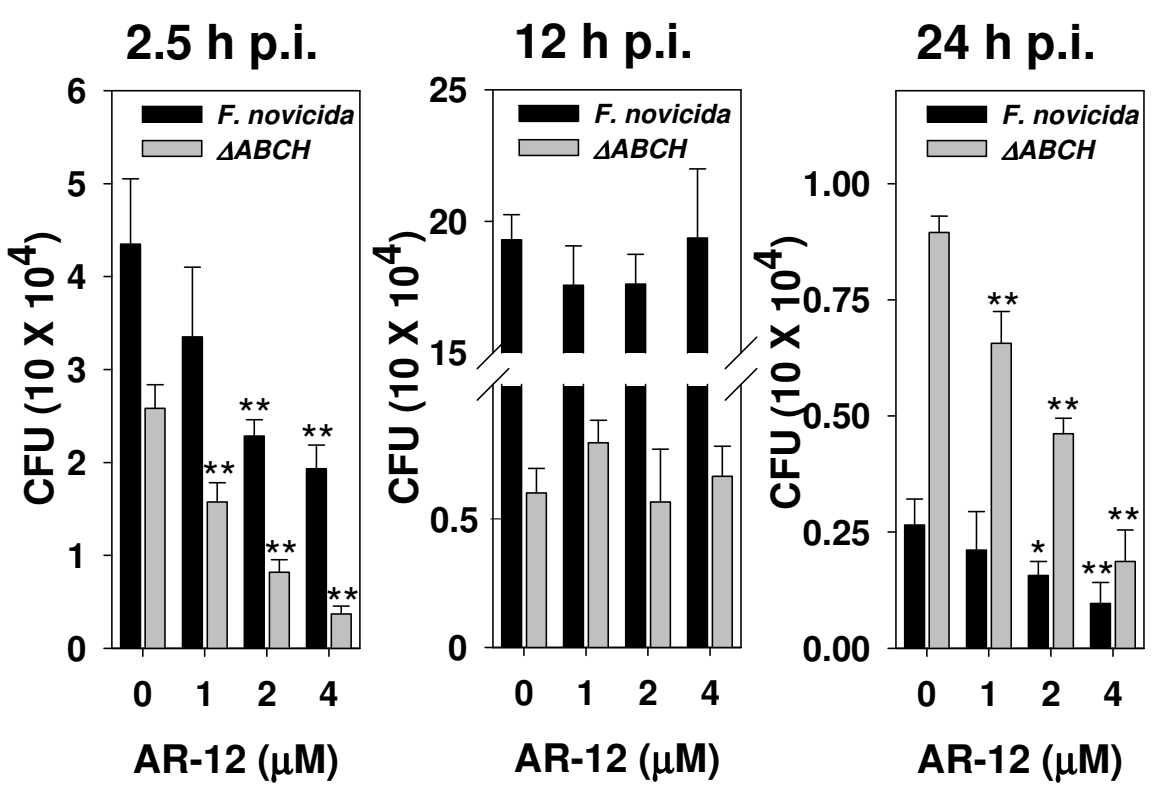

\section{Figure 4}

Susceptibility of intracellular F. novicida to AR-I 2 varies at different time points post-infection. THP-I macrophages were infected with $F$. novicida (dark bar) or a quadruplicate acid phosphatase deletion mutant $(\triangle \mathrm{ABCH}$; gray bar) and treated with various doses of AR-I 2 for $3 \mathrm{~h}$ beginning at different time points post-infection. The number of surviving intracellular bacteria was determined by the CFU assay. Columns, mean; bars, $\pm S D(n=3)$. $* P<0.05$, $* * P<0.0$ l. 
ication by inhibiting the activation of autophagy with bacterial secretory proteins [32-34]. The findings presented here indicate that intracellular $F$. tularensis at $12 \mathrm{~h}$ postinfection is not susceptible to the antibacterial activity of AR-12; in contrast to the susceptibility seen at $2.5 \mathrm{~h}$ and $24 \mathrm{~h}$ post-infection. Since AR-12-induced killing of intracellular Francisella is mediated primarily through an autophagy-dependent mechanism, we speculate that bacteria evade the antibacterial effect of AR-12 by inhibiting autophagy activation at $12 \mathrm{~h}$ post-infection. This notion is supported by evidence showing that intracellular infection with Francisella down-regulates several autophagyrelated genes in THP-1 human monocytes, including ATG5, ATG12, ATG16L2, ATG7, ATG4A and class III PI3K $[35,36]$. Although these reported data were obtained at 24 $\mathrm{h}$ post-infection, we cannot exclude the possibility that this down-regulation of autophagy by Francisella occurs at an earlier stage of infection such as after phagocytosis and exposure to the phagosomal environment. Moreover, our findings from the use of the quadruplicate acid phosphatase mutant strain $(\triangle \mathrm{ABCH})$ indicate that this resistance to AR-12 involves pathways affecting autophagy or other aspects of host resistance that are independent of the intracellular location of the organism. Nonetheless, the fact that intracellular Francisella are located within autophagosomes at late stages of infection suggest that this autophagy inhibitory activity of the bacteria declines, resulting in restoration of susceptibility to autophagic eradication and rendering them sensitive to the antibacterial activity of AR-12.

AR-12 has been reported to inhibit a variety of cellular enzymes, including PDK-1 and P21 activated kinase-1 (PAK-1), and to induce endoplasmic reticulum (ER) stress [37-39]. Among these activities, the induction of ER stress has been shown to contribute to the AR-12-induced activation of autophagy in cancer cells for which the activity of PKR-like ER kinase (PERK) was determined to be important [26]. Activation of PERK, considered to be the central regulatory kinase for the unfolded protein response in eukaryotic cells, leads to autophagosome formation through the phosphorylation eIF2 $\alpha$ and subsequent formation of the Atg5-Atg12(-Atg6) complex [40,41]. Although important for AR-12-induced autophagy in cancer cells, it is not known whether PERK has a similar role in infected macrophages. Moreover, no evidence to date has shown that AR-12 interacts directly with PERK leading to its activation. Indeed, AR-12 has been shown to inhibit the activities of PDF-1 and PAK-1 through interaction with their kinase domains, suggesting that the activation of PERK activity is an indirect effect of the drug. Based on the reported activities of celecoxib, the compound from which AR-12 was derived, other potential targets of AR-12 include carbonic anhydrase, sarcoplasmic/ER calcium ATPase (SERCA), COX-1 and COX-2
[42]. Among these enzymes, SERCA is interesting in that it plays an important role in regulating $\left[\mathrm{Ca}^{2+}\right]$ in the ER. Inhibition of SERCA will cause $\mathrm{Ca}^{2+}$ efflux from ER to the cytosol, leading to the unfolded protein response and subsequent activation of PERK. Although there is no direct evidence showing that AR-12 can interact with SERCA, the cytosolic $\left[\mathrm{Ca}^{2+}\right]$ of cancer cells increases immediately after exposure to AR-12 which suggests that AR-12 may inhibit SERCA activity [31]. Identification of the mechanism of how AR-12 induces autophagy should facilitate the development of more potent and specific host cell-targeted antibacterial agents.

We recently reported that celecoxib, the parent compound of AR-12, also exhibits anti-Francisella activity, and that subsequent screening of a celecoxib-based focused compound library identified Compound 20, a novel analog with multi-fold greater antibacterial activity [31]. Unlike AR-12, however, Compound 20 showed direct growthinhibitory activity against $F$. novicida and $F$. tularensis (Schu S4, a type A strain) at low $\mu \mathrm{M}$ concentrations. While the intracellular growth of Francisella was also inhibited by Compound 20, this occurred at concentrations that far exceeded those shown for AR-12. Although the antibacterial target of Compound 20 has not been identified, these differences in activity suggest that Compound 20 and AR12 represent two novel classes of compound with distinct antibacterial mechanisms. These findings highlight the potential value of existing drugs as important sources of lead compounds for the development of novel antibacterial agents.

\section{Competing interests}

The authors declare that they have no competing interests.

\section{Authors' contributions}

HCC conceived of the study, designed and performed most experiments, and drafted the manuscript. SS conducted the experiment with virulent Francisella tularensis strain Schu S4. SKK helped to design experiment and revised the manuscript. HC helped the assay of intracellular Francisella novicida in macrophage. DW conducted the synthesis of AR-12. JSG provided the $\triangle \mathrm{ABCH}$ strain of $F$. novicida and helped to design experiments. LSS helped to design experiments and revised the manuscript. CSC conceived of the study and revised the manuscript. All authors read and approved the final manuscript.

\section{Acknowledgements}

This work was supported by the NIH/NIAID Regional Center of Excellence for Bio-defense and Emerging Infectious Diseases Research (RCE) Program and the Lucius A. Wing Endowed Chair Fund (to CSC). The authors wish to acknowledge membership within and support from the Region V 'Great Lakes' RCE (NIH award I-U54-AI-057I53). 


\section{References}

I. Nakatogawa H, Suzuki K, Kamada Y, Ohsumi Y: Dynamics and diversity in autophagy mechanisms: lessons from yeast. Nat Rev Mol Cell Biol 2009, I 0:458-467.

2. Orvedahl A, Levine B: Eating the enemy within: autophagy in infectious diseases. Cell Death Differ 2009, I 6:57-69.

3. Gutierrez MG, Master SS, Singh SB, Taylor GA, Colombo MI, Deretic $\mathrm{V}$ : Autophagy is a defense mechanism inhibiting BCG and Mycobacterium tuberculosis survival in infected macrophages. Cell 2004, I I 9:753-766.

4. Nakagawa I, Amano A, Mizushima N, Yamamoto A, Yamaguchi $H$, Kamimoto T, Nara A, Funao J, Nakata M, Tsuda K, Hamada S, Yoshimori $\mathrm{T}$ : Autophagy defends cells against invading group $\mathbf{A}$ Streptococcus. Science 2004, 306:1037-1040.

5. Birmingham CL, Smith AC, Bakowski MA, Yoshimori T, Brumell JH: Autophagy controls Salmonella infection in response to damage to the Salmonella-containing vacuole. J Biol Chem 2006, 28 I: I I374-I I383.

6. Ellis J, Oyston PC, Green M, Titball RW: Tularemia. Clin Microbiol Rev 2002, I 5:631-646.

7. Oyston PC: Francisella tularensis: unravelling the secrets of an intracellular pathogen. J Med Microbiol 2008, 57:921-930.

8. Sjostedt A: Virulence determinants and protective antigens of Francisella tularensis. Curr Opin Microbiol 2003, 6:66-7I.

9. Dennis DT, Inglesby TV, Henderson DA, Bartlett JG, Ascher MS, Eitzen E, Fine AD, Friedlander AM, Hauer J, Layton M, Lillibridge SR, McDade JE, Osterholm MT, O'Toole T, Parker G, Perl TM, Russell PK, Tonat K: Tularemia as a biological weapon: medical and public health management. Jama 200I, 285:2763-2773.

10. Oyston PC, Sjostedt A, Titball RW: Tularaemia: bioterrorism defence renews interest in Francisella tularensis. Nat Rev Microbiol 2004, 2:967-978.

II. Alibek K: Biohazard New York, NY: Random House; 1999.

12. Clemens DL, Lee BY, Horwitz MA: Virulent and avirulent strains of Francisella tularensis prevent acidification and maturation of their phagosomes and escape into the cytoplasm in human macrophages. Infect Immun 2004, 72:3204-32 I 7.

13. Santic M, Asare R, Skrobonja I, Jones S, Abu Kwaik Y: Acquisition of the vacuolar ATPase proton pump and phagosome acidification are essential for escape of Francisella tularensis into the macrophage cytosol. Infect Immun 2008, 76:267I-2677.

14. Mohapatra NP, Balagopal A, Soni S, Schlesinger LS, Gunn JS: AcpA is a Francisella acid phosphatase that affects intramacrophage survival and virulence. Infect Immun 2007, 75:390-396.

15. Mariathasan S, Weiss DS, Dixit VM, Monack DM: Innate immunity against Francisella tularensis is dependent on the ASC/caspase-I axis. J Exp Med 2005, 202: I043-1049.

16. Lai $\mathrm{XH}$, Golovliov I, Sjostedt A: Francisella tularensis induces cytopathogenicity and apoptosis in murine macrophages via a mechanism that requires intracellular bacterial multiplication. Infect Immun 2001, 69:4691-4694.

17. Checroun C, Wehrly TD, Fischer ER, Hayes SF, Celli J: Autophagymediated reentry of Francisella tularensis into the endocytic compartment after cytoplasmic replication. Proc Natl Acad Sci USA 2006, I 03: |4578-|4583.

18. Mohapatra NP, Soni S, Reilly TJ, Liu J, Klose KE, Gunn JS: Combined deletion of four Francisella novicida acid phosphatases attenuates virulence and macrophage vacuolar escape. Infect Immun 2008, 76:3690-3699.

19. Santic M, Akimana C, Asare R, Kouokam JC, Atay S, Kwaik YA: Intracellular fate of Francisella tularensis within arthropodderived cells. Environ Microbiol 2009, I I(6): |473-8I.

20. Zhu J, Huang J-W, Tseng P-H, Yang Y-T, Fowble J, Shiau C-W, Shaw Y-J, Kulp SK, Chen C-S: From the Cyclooxygenase-2 Inhibitor Celecoxib to a Novel Class of 3-Phosphoinositide-Dependent Protein Kinase-I Inhibitors. Cancer Res 2004, 64:4309-43 I 8.

21. Balagopal A, MacFarlane AS, Mohapatra N, Soni S, Gunn JS, Schlesinger LS: Characterization of the receptor-ligand pathways important for entry and survival of Francisella tularensis in human macrophages. Infect Immun 2006, 74:5 I I 4-5I 25.

22. Gavrilin MA, Bouakl IJ, Knatz NL, Duncan MD, Hall MW, Gunn JS, Wewers MD: Internalization and phagosome escape required for Francisella to induce human monocyte IL-I beta processing and release. Proc Natl Acad Sci USA 2006, I 03: |4|-| 46.
23. Jeffrey M, Edmondson LSAaAOM: A rapid and simple MTT-based spectrophotometric assay for determining drug sensitivity in monolayer cultures. Methods in Cell Science 1988, I I (I):3.

24. Racher AJ, Looby D, Griffiths JB: Use of lactate dehydrogenase release to assess changes in culture viability. Cytotechnology 1990, 3:301-307.

25. Park MA, Curiel DT, Koumenis C, Graf M, Chen CS, Fisher PB, Grant $S$, Dent P: PERK-dependent regulation of HSP70 expression and the regulation of autophagy. Autophagy 2008, 4:364-367.

26. Park M, Yacoub A, Rahmani M, Zhang G, Hart L, Hagan M, Calderwood S, Sherman M, Koumenis C, Spiegel S, Chen CS, Graf M, Curiel $D$, Fisher P, Grant S, Dent P: OSU-030I2 stimulates PERKdependent increases in HSP70 expression, attenuating its lethal actions in transformed cells. Mol Pharmacol 2008, 73: II68-II84.

27. Gao M, Yeh PY, Lu YS, Hsu CH, Chen KF, Lee WC, Feng WC, Chen CS, Kuo ML, Cheng AL: OSU-030I 2 a novel celecoxib derivative, induces reactive oxygen species-related autophagy in hepatocellular carcinoma. Cancer Res 3012, 68:9348-9357.

28. Chiu HC, Kulp SK, Soni S, Wang D, Gunn JS, Schlesinger LS, Chen CS: Eradication of intracellular Salmonella enterica serovar Typhimurium with a small-molecule, host cell-directed agent. Antimicrob Agents Chemother 2009, 53:5236-5244.

29. Finlay $B B$, Hancock RE: Can innate immunity be enhanced to treat microbial infections? Nat Rev Microbiol 2004, 2:497-504.

30. Weng SC, Kashida Y, Kulp SK, Wang D, Brueggemeier RW, Shapiro $C L$, Chen CS: Sensitizing estrogen receptor-negative breast cancer cells to tamoxifen with OSU-030 I 2 a novel celecoxibderived phosphoinositide-dependent protein kinase-I/Akt signaling inhibitor. Mol Cancer Ther 30I2, 7:800-808.

31. Wang YC, Kulp SK, Wang D, Yang CC, Sargeant AM, Hung JH, Kashida Y, Yamaguchi M, Chang GD, Chen CS: Targeting endoplasmic reticulum stress and Akt with OSU-030I2 and gefitinib or erlotinib to overcome resistance to epidermal growth factor receptor inhibitors. Cancer Res 2008, 68:2820-2830.

32. Cullinane M, Gong L, Li X, Lazar-Adler N, Tra T, Wolvetang E, Prescott M, Boyce JD, Devenish RJ, Adler B: Stimulation of autophagy suppresses the intracellular survival of Burkholderia pseudomallei in mammalian cell lines. Autophagy 2008, 4:744-753.

33. Ogawa M, Yoshimori T, Suzuki T, Sagara H, Mizushima N, Sasakawa C: Escape of Intracellular Shigella from Autophagy. Science 2005, 307:727-731.

34. Amer AO, Swanson MS: Autophagy is an immediate macrophage response to Legionella pneumophila. Cell Microbiol 2005, 7:765-778.

35. Butchar JP, Cremer TJ, Clay CD, Gavrilin MA, Wewers MD, Marsh CB, Schlesinger LS, Tridandapani S: Microarray analysis of human monocytes infected with Francisella tularensis identifies new targets of host response subversion. PLOS ONE 2008, 3:e2924.

36. Cremer TJ, Amer A, Tridandapani S, Butchar JP: Francisella tularensis regulates autophagy-related host cell signaling pathways. Autophagy 2009, 5:125-128.

37. Zhu J, Huang JW, Tseng PH, Yang YT, Fowble J, Shiau CW, Shaw YJ, Kulp SK, Chen CS: From the cyclooxygenase-2 inhibitor celecoxib to a novel class of 3-phosphoinositide-dependent protein kinase-I inhibitors. Cancer Res 2004, 64:4309-43 I8.

38. Porchia LM, Guerra M, Wang YC, Zhang Y, Espinosa AV, Shinohara M, Kulp SK, Kirschner LS, Saji M, Chen CS, Ringel MD: 2-amino-N\{4-[5-(2-phenanthrenyl)-3-(trifluoromethyl)- I H-pyrazol- Iyl]-phe nyl\} acetamide (OSU-030I2), a celecoxib derivative, directly targets p2I-activated kinase. Mol Pharmacol 2007, 72: I I24-II3I.

39. Yacoub A, Park MA, Hanna D, Hong Y, Mitchell C, Pandya AP, Harada H, Powis G, Chen CS, Koumenis C, Grant S, Dent P: OSU-03012 promotes caspase-independent but PERK-, cathepsin B-, BID-, and AIF-dependent killing of transformed cells. Mol Pharmacol 2006, 70:589-603.

40. Ogata M, Hino S, Saito A, Morikawa K, Kondo S, Kanemoto S, Murakami T, Taniguchi M, Tanii I, Yoshinaga K, Shiosaka S, Hammarback JA, Urano F, Imaizumi K: Autophagy is activated for cell survival after endoplasmic reticulum stress. Mol Cell Biol 2006, 26:9220-9231.

4I. Kouroku Y, Fujita E, Tanida I, Ueno T, Isoai A, Kumagai H, Ogawa S, Kaufman RJ, Kominami E, Momoi T: ER stress (PERK/elF2alpha phosphorylation) mediates the polyglutamine-induced LC3 
conversion, an essential step for autophagy formation. Cell Death Differ 2007, I 4:230-239.

42. Schonthal AH: Direct non-cyclooxygenase-2 targets of celecoxib and their potential relevance for cancer therapy. Br J Cancer 2007, 97: | 465- I 468.

Publish with Bio Med Central and every scientist can read your work free of charge

"BioMed Central will be the most significant development for disseminating the results of biomedical research in our lifetime. " Sir Paul Nurse, Cancer Research UK

Your research papers will be:

- available free of charge to the entire biomedical community

- peer reviewed and published immediately upon acceptance

- cited in PubMed and archived on PubMed Central

- yours - you keep the copyright

Submit your manuscript here:

http://www.biomedcentral.com/info/publishing_adv.asp
BioMedcentral 